\title{
Extraction of soil moisture from RADARSAT-1 and its role in the formation of the 6 December 2008 landslide at Bukit Antarabangsa, Kuala Lumpur
}

\begin{abstract}
Active microwave has a huge potential in the estimation of soil moisture especially over large areas where the meteorological observations are seldom. The large contrast in dielectric constant between different types of soil is considered as the main factor for measuring the moisture content. This study is aimed at the extraction of soil moisture over the areas of Bukit Antarabangsa, Malaysia using active microwave remote sensing technique in order to examine the impact of moisture content dynamically on landslides occurrence, which have been a basic challenge that threaten Bukit Antarabangsa area, particularly in falling of monsoon seasons. This study addressed a specific event that took place in 6 December 2008 due to a very high level of precipitation that resulted in a raise in ground water table causing the occurrence of landslide. One Radarsat-1 image acquired in July 2008 before the landslide was used for generating the moisture content map. The resultant moisture content map showed a reasonable distribution of the moisture concentrated over the forest areas which has previous records landslides. Moreover, it was found that the previous landslide events were within the high moisture zone indicating the presence of high moisture content. Subsequently, three moisture maps were extracted from Landsat-7 ETM+, which were then used for validation process. A statistically based validation technique was used by calculating area under the curve that correlates the high moisture values of three images. In order to validate the Landsat-7 ETM+ moisture content, monthly rainfall data was plotted against the high moisture values derived from three Landsat-7 images. The validation result indicated an acceptable compatibility. The spatial relation between high moisture areas in Landsat-7 ETM+ images along the year resulted in a good fitting in the highï low moisture distribution areas with sensitivity ranged of 6010 i $70 \%$. Finally, the moisture content map generated by Radarsat-1 was validated using a landslide inventory map. The resultant validation produced an area under curve of $0.704(70 \%)$.
\end{abstract}

Keyword: Landslides; Malaysia; Moisture content; Radarsat-1; Remote sensing 